\title{
ULTIMATE RUIN PROBABILITIES FOR GENERALIZED GAMMA-CONVOLUTIONS CLAIM SIZES
}

\author{
BY \\ M. USÁBEL \\ Universidad Carlos III de Madrid
}

\begin{abstract}
A method of inverting the Laplace transform based on the integration between zeros technique and a simple acceleration algorithm is presented. This approach was designed to approximate ultimate ruin probabilities for $\Gamma$-convolutions claim sizes, but it can be also used with other distributions. The stable algorithm obtained yields interval approximations (lower and upper bounds) to any desired degree of accuracy even for very large values of $u(1,000,000)$, initial reserves, without increasing the number of computations. This last fact can be considered an interesting property compared with other recursive methods previously used in actuarial literature or other methods of inverting Laplace transforms.
\end{abstract}

\section{KEYWORDS AND PHRASES}

Ultimate ruin probability, upper and lower bounds, stable recursive algorithms, numerical inversion of the Laplace transform.

\section{INTRODUCTION}

Let us consider the Classical risk process in continuous time $\left\{Z_{t}\right\}_{t>0}$ with $U_{k}$ claim sizes and premium $\rho$ per time unit,

$$
Z_{t}=u+\rho t-\sum_{k=1}^{N_{t}} U_{k}
$$

where $u$ are the initial reserves and $N_{t}$ the total number of claims up to time $t$ following a homogeneous Poisson process of parameter $\lambda>0$. Let $F(x)$ denote the distribution function of claim sizes $U_{k}$ with mean $p_{1}$ and $p=\lambda p_{1}(1+\theta)$, where $\theta$ is the premium loading factor. We will also assume that $\theta>0$ and $F(x)=0$.

Using a renewal argument and Theorem 13.5.1 of the text-book by Bowers et al. (1997), the ultimate non-ruin probability can be expressed 
using the following integral equation (Volterra integral equation of the second kind)

$$
\Phi(u)=\frac{\theta}{(1+\theta)}+\frac{1}{(1+\theta) p_{1}} \int_{0}^{u} \Phi(u-w)(1-F(w)) d w
$$

or in the case of ruin probability, see Gerber $(1979$, p. 115 , equation $(3.7)$

$$
\Psi(u)=\frac{1}{(1+\theta) p_{1}} A(u)+\frac{1}{(1+\theta) p_{1}} \int_{0}^{u} \Phi(u-w)(1-F(w)) d w
$$

where

$$
A(w)=\int_{w}^{\infty}(1-F(z)) d z
$$

Since the early 1980 s, many methods have been developed in order to approximate $\Psi(u)$. They were based on a discretization of some aspect of the risk process and derived recursive expressions; see for example Goovaerts and De Vylder (1984), Panjer (1986), Dickson (1989), Dickson and Waters (1991), Ramsay (1992b), Dickson, Egidio dos Reis and Waters (1995). Panjer and Wang (1993) describe the conditions under which these recursions are stable.

Although some of these recursive approaches may be able to determine $\Psi(u)$ to any desired degree of accuracy, they may not be suitable for heavytailed distributions, such as the Pareto or lognormal distribution for two reasons, citing Ramsay and Usábel (1997):

1. To achieve a reasonable degree of accuracy, the interval of discretization must be at most one unit of the mean of length. If we standardize the unit of currency such that $p_{1}=1$, then to obtain $\Psi(10)$ we must recursively estimate every intermediate unit point $\Psi(u)$ for $k=0,1,2, \ldots, 9,10$. This may be acceptable if we need only small values of $u$; however, for large values of $u$, say $u=500$ units, this method can be slow. For the Pareto, $\Psi(500)$ is not insignifcant.

2. The quadrature rules inherent in the recursive schemes are usually of low order. This further reduces its accuracy and its rate of convergence. To improve accuracy, the intervals of discretization are made even smaller. This substantially increases the number of intermediate calculations required, making the process of finding $\Psi(u)$ slower.

The above presented problem was partially solved using product integration by Ramsay and Usábel (1997); where it was proved that the convergence of the method was significantly faster than former methods of actuarial literature. Nevertheless, in this method, accuracy is also eventually menaced by increasing values of the initial reserves and the convergence is of order $O\left(h^{2}\right)$ and the true errors are not easily estimated.

Before the shift to recursive methods explained in the last paragraph, the problem of ruin in the Collective Risk Theory had been extensively treated in actuarial literature using integral transforms.

Since the paper by Sparre Andersen in 1955 many authors developed approximations for the ruin probability using Laplace-Stieltjes transforms. 
Cramér (1955) used the Winer-Höpf method for the classical case and Thorin $(1970,71,77)$ introduced the generalization when epochs of claims form a renewal process. Thorin and Wikstad $(1971,73,77)$ used Piessens (1969) inversion method of the Laplace transforms and Bohman $(1971,74,75)$, focussed on inversions of Fourier transforms and Seal $(1971,74)$ dealt with both Laplace and Fourier numerical inversions. Seal (1977) obtained an interesting result for the classical case and exponential claim size distribution using the Bromwich-Mellin inversion formula for Laplace transforms.

Numerical illustrations obtained using this methodology were based on Laplace transforms inversion techniques due to Piessens (1969, 71). These methods can be considered very accurate in the cases contemplated but the theoretical error is not easy to control. In this context, we should mention the very much cited approach, and commonly used in non-life insurance practice in North America, presented by Heckman and Meyers (1983). Other works on ruin probability approximations are Gerber, Goovaerts and Kaas (1987); Ramsay (1992a); Cai and Garrido (1998) and Usábel (1999).

The study of the tail probabilities of the stationary waiting times is the counterpart concept in the context of queueing systems. Many interesting works were presented in this field dealing with long-tailed distributions such as Choudhury, Gupta and Agarwal (1992); Abate, Choudhury and Whitt (1994); Abate, Choudhury and Whitt (1995); Glynn and Whitt (1995). Some of them were specially focussed on the use of Laplace of Fourier transforms as Abate and Whitt (1992, 95, 96) and Choudhury and Whitt (1997). Finally, some interesting asymptotic approximations were produced by Embrechts and Veraverbeke (1982); Willekens and Teugels (1992) and Abate, Choudhury and Whitt (1994).

The study of the Laplace transform of the ultimate ruin probability when claim sizes follow a generalized $\Gamma$-convolution function is contained in section 2 . Some of the most frequently used heavy-tailed distributions in actuarial science belongs to this family. Thorin (1977a) or Berg (1981) proved that Pareto distributions are members of this family; so Thorin (1977 b) did with Log-normal distributions. Other outstanding works related with $\Gamma$-convolution functions are Thorin (1978) and Goovaerts, D'Hooge and De Pril (1977).

In the present work, a method of inverting Laplace transforms based on the integration between zeros method (along with an acceleration algorithm: a generalization of the Euler method) is introduced when approximating ultimate ruin probabilities in the Classical case of risk theory. We will show that it is specially recommended for large values of $u$ and heavy-tailed distributions.

In sections 3, 4 and 5 we will lay the theoretical foundations to consider the integration between zeros technique an interesting approach when solving integral (3.2) and, subsequently, obtaining interval approximations (lower and upper bounds) for the ultimate ruin probability function when claim sizes are $\Gamma$-convolution functions. In section 6 , the use of the mid-point integration technique or three point trapezoidal rule will be proved to reduce drastically the number of calculations involved. Section 7 is devoted to asymptotic results for large values of the initial reserves $u$. Finally, numerical examples are presented in section 8 , confirming the promised efficiency explained in the theoretical results. 
2. THE LAPLACE TRANSFORM OF THE ULTIMATE RUIN PROBABILITY FOR GENERALIZED $\Gamma$-CONVOLUTION CLAIM SIZE

Using the Laplace transform on expression (1.1)

$$
\begin{aligned}
\Phi^{*}(s) & =\frac{\frac{\theta}{1+\theta}\left(\frac{1}{s}\right)}{1-\frac{1}{(1+\theta) p_{1}}\left(\frac{1}{s}-F^{*}(s)\right)} \\
& =\frac{\frac{\theta}{1+\theta}}{s-\left(\frac{1}{(1+\theta) p_{1}}\right)+\left(\frac{1}{(1+\theta) p_{1}}\right) f^{*}(s)}
\end{aligned}
$$

where $f(x)$ is the d.f. of the claim size distribution and,

$$
f^{*}(s)=\int_{0}^{+\infty} f(x) e^{-s x} d x=s F^{*}(s)
$$

It is obvious that the ruin probability

$$
\Psi(u)=1-\Phi(u)
$$

has the following Laplace transform

$$
\Psi^{*}(s)=\frac{1}{s}-\Phi^{*}(s)
$$

A distribution function defined on the non-negative real axis is a generalized $\Gamma$-convolution if its Laplace transform can be written

$$
f^{*}(s)=\int_{0}^{+\infty} f(x) e^{-s x} d x=e^{-a s} e^{\int_{0}^{\infty} \ln \left(\frac{1}{1+\left(\frac{s}{y}\right)}\right) d U(y)} \operatorname{Re}(s) \geq 0
$$

where $a \geq 0$ and $U(y)$ is nondecreasing and such that

$$
\begin{gathered}
U(0)=0 \\
\int_{0}^{1}|\ln (y)| d U(y)<\infty \\
\int_{0}^{\infty} \frac{d U(y)}{y}<\infty
\end{gathered}
$$

Some of the most frequently used heavy-tailed distributions in actuarial science belongs to this family. Thorin (1977) or Berg (1981) proved that Pareto distributions are members of this family with parameters $a=0$ and

$$
U^{\prime}(y)=-\frac{1}{\pi} \frac{q^{\prime}(y)}{1+(q(y))^{2}} \quad y>0
$$


and

$$
q^{\prime}(y)=\frac{\Gamma(1+h)}{\pi} e^{g y} g(g x)^{-h-1}
$$

where the Pareto c.d.f. with parameters $g$ and $h$ was defined as

$$
F(x)=1-\left(\frac{x}{g}\right)^{-h} \quad x \geq g
$$

Thorin (1977) also proved the same condition for Log-normal c.d.fs. with parameters $a$ and $\beta$

$$
\Lambda(x)=N\left(\frac{\log (a x)}{\beta}\right) \quad x>0
$$

obtaining that, again in this case, $a=0$ and

$$
U(y)=\frac{1}{\pi} \operatorname{arctg} \frac{\operatorname{Im} \lambda^{+}(y)}{\operatorname{Re} \lambda^{+}(y)}
$$

where

$$
\lambda^{+}(y)=\frac{e^{\pi^{2} /\left(2 / \beta^{2}\right)}}{\sqrt{2 \pi}} \int_{-\infty}^{\infty} \exp \left(-\frac{x}{a} e^{-\beta u}-\frac{1}{2} u^{2}+\frac{I \pi u}{\beta}\right) d u
$$

and

$$
I=\sqrt{-1}
$$

The following theorem (proved in the appendix) will be most interesting for future developments,

Theorem 1. For $c>0$ and $z \geq 0$, the real part of the Laplace transform of the ruin probability for $\Gamma$-convolution function claim size, $\operatorname{Re}\left(\Psi^{*}(c+z I)\right)$

1. is asymptotically close to 0 with increasing values of $z$.

$$
\lim _{z \rightarrow \infty} \operatorname{Re}\left(\Psi^{*}(c+z I)\right)=0
$$

2. is always bound and smooth.

\section{THE INTEGRATION BETWEEN ZEROS APPROACH}

In order to obtain the inverse Laplace transform we can use the BromwichMellin inversion formula,

$$
\Psi(u)=\frac{1}{2 \pi I} \int_{c^{-\infty i}}^{c+\infty i} e^{s u} \Psi^{*}(s) d s
$$

where $c$ is a positive real constant that exceeds the real part of all singularities of $\Psi^{*}(s)$. 
Unfortunately, when $f(x)$ is a generalized $\Gamma$-convolution function, $\Psi^{*}(s)$ has no isolated singular points because $f^{*}(s)$ is not defined for $\operatorname{Re}(s)<0$ and we proved in Lemma 1 a) that $\Psi^{*}(s)$ is always bounded for $\operatorname{Re}(s) \geq 0$. This last fact means that we cannot benefit, for instance, from Residues Theorem in order to approximate $\Psi(u)$ using its Laplace transform. sion

Within the actuarial literature, Seal (1977) proposed the following expres-

$$
\Psi(u)=\frac{2 e^{c u}}{\pi} \int_{0}^{\infty} \operatorname{Re}\left(\Psi^{*}(c+z I) \cos (u z) d z\right.
$$

and Heckman and Myers (1983) used an alternative formula based on the results by Kendall and Stuart (1977)

$$
\Psi(u)=\frac{1}{2}+\frac{1}{\pi} \int_{0}^{\infty} \frac{\bmod \left(\Psi^{*}(z / \sigma)\right)}{z} \sin \left(\frac{z u}{s}-\arg \left(\Psi^{*}(z / \sigma)\right)\right) d z
$$

where mod and arg are the modulus and argument functions respectively.

It is not an easy task solving (3.2) or (3.3) numerically because the integrand is a rapidly oscillating function. As $z \rightarrow \infty$ we will face plus areas and minus areas of nearly equal size and the resulting cancellation of area is attended by a loss of significance, specially when $\lim _{z \rightarrow \infty} \operatorname{Re}\left(\Psi^{*}(c+z I)\right)=0$ (see figure 1).

Heckman and Meyers (1983) already used the integration between zeros in order to approximate integral (3.3) to assess the total claims distributions. Davies and Rabinowitz (1984) also cited the integration between zeros as a valuable method in approximating these integrals. They all argued that it is advantageous to use a rule that employs the values of the integrand at the endpoints of the integration intervals. Since the integrand is zero at these points, more accuracy is obtained without additional computation.

We should, nevertheless, point out that the formula (3.3) used by Heckman and Myers (1983) is not very suitable for fully exploiting the advantages of the integration between zeros to numerically approximate the ultimate ruin probability for $\Gamma$-convolutions claim size. The main reasons argued are

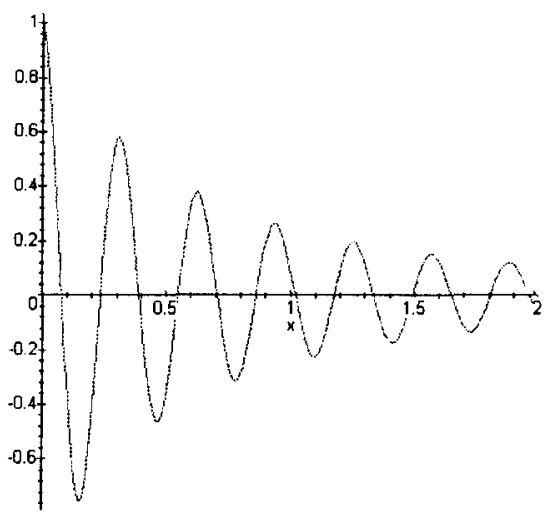

Figure 1 
1. The formula uses the parameter $\sigma$ that must be obtained from the second order moment of the claim size distribution. The heaviest-tailed members of the $\Gamma$-convolutions claims size family may have no such moment.

2. The basic interval length, $h=2 \pi \sigma /$ (maximum claim amount), cannot be defined for claim size distributions with support in all the positive real axis (see p. 40 of the original work for further details).

Let us now review again this methodology on solving (3.2). It is very easy to prove that

$$
\begin{aligned}
\Psi(u)= & \frac{2 e^{u c}}{\pi} \int_{0}^{\frac{\pi}{2 u}} \operatorname{Re}\left(\Psi^{*}(c+z I) \cos (u z) d z\right. \\
& +\frac{2 e^{u c}}{\pi} \sum_{i=1}^{\infty} \int_{\frac{\pi}{2 u}+\frac{\pi}{2 u}+\frac{i \pi}{u}}^{\frac{(i-1) \pi}{u}} \operatorname{Re}\left(\Psi^{*}(c+z I)\right) \cos (u z) d z \\
= & \sum_{i=0}^{\infty} a_{i}(u)
\end{aligned}
$$

where

$$
\begin{aligned}
a_{0}(u) & =\frac{2 e^{u c}}{\pi} \int_{0}^{\frac{\pi}{2 u}} \operatorname{Re}\left(\Psi^{*}(c+z I)\right) \cos (u z) d z \\
a_{i}(u) & =\frac{2 e^{u c}}{\pi} \int_{\frac{\pi}{2 u}+\frac{(i-1) \pi}{u}}^{\frac{\pi}{2 u}+\frac{i \pi}{u}} \operatorname{Re}\left(\Psi^{*}(c+z I)\right) \cos (u z) d z \\
i & =1,2, \ldots
\end{aligned}
$$

Approximating integral (3.2) using the series formula above, as many other numerical techniques, generates two types of errors

1. Discretization error $\left(\varepsilon^{d}(u)\right)$.

2. Truncation error $\left(\varepsilon_{n}(u)\right)$.

\section{THE DISCRETIZATION ERROR $\left(\varepsilon^{d}(u)\right)$}

It is obvious that the family of integrals $\left\{a_{i}(u)\right\}_{i=0}^{\infty}$ in most cases, shall be evaluated numerically and the errors must be considered. The discretization total error then will be the sum of all of them. Obviously, the stability of the method fully relies on the numerical technique used when approximating the mentioned integrals and not on other more complicated considerations. Numerical analysis libraries can certainly provide us with stable, fast and accurate approximations algorithms in order to obtain these integrals.

It is very plain to see that the reduction of the dicretization total error will be paid in terms of more evaluations of the function $\operatorname{Re}\left(\Psi^{*}(c+z l)\right)$ within the intervals $z \in\left(\frac{\pi}{2 u}+\frac{(i-1) \pi}{u}, \frac{\pi}{2 u}+\frac{i \pi}{u}\right)$ and the subsequent increase on the computation times.

We should be fully aware that large values of the initial reserves, $u$, will generate tighter intervals with a positive effect on the discretization error. 


\section{THE TRUNCATION ERROR $\left(\varepsilon_{n}(u)\right)$}

Using (3.4), it is clear that the sequence $\left\{a_{i}(u)\right\}_{i=0}^{\infty}$ generates the approximations $\left\{S_{n}(u)\right\}$

$$
S_{n}(u)=\sum_{i=0}^{n} a_{i}(u)
$$

It is obvious that the larger the $n$ considered the more accuracy and the more computations required.

Nevertheless, the order of convergence of the initial sequence ought not to be necessarily large and, subsequently, this approach would not be very efficient when seeking for a high precision approximation. As it is suggested by Davies and Rabinowitz (1984), many extrapolations techniques were proved to work effectively in accelerating the convergence of sequences of partial sums of series resulting from the integration of oscillatory integrals. These authors cited the Euler transformation, or the variation introduced by Longman (1956), Richardson's extrapolation, the $\varepsilon$-transformation, iterations of Aitken's $\Delta^{2}$ method, Levin's V-transformation (Levin (1973) or the work by van de Vooren and van Linde (1966). For a survey of extrapolation processes in numerical analysis see Joyce (1971).

However, it is not proved that the mentioned standard extrapolation techniques applied to the former sequences will yield upper and lower limits. Actually, most of them cannot guarantee this fact. Moreover, these techniques, although useful in some cases, are not proved to accelerate the convergence in many others.

We will now show how the convergence can be substantially accelerated using a simple generalization of the Euler extrapolation technique obtaining upper and lower bounds sequences and decreasing errors.

With the following theorem (proved in the appendix), we will now show why integration between zeros, used on formula (3.2), is most interesting when approximating ultimate ruin probabilities for generalized $\Gamma$-convolution claim sizes.

Theorem 2. When the ultimate ruin probability can be expressed as the alternating series

$$
\Psi(u)=\sum_{i=0}^{\infty}(-1)^{i} a_{i}(u)
$$

where $\left\{a_{i}(u)\right\}_{i=0}^{\infty}$ is a bound and smooth sequence for which $\lim _{i \rightarrow \infty} a_{i}(u)=0$, the following equality holds

$$
\Psi(u)=A^{j}(u)+\left(\frac{(-1)^{j}}{2^{j}}\right) \sum_{i=0}^{\infty}(-1)^{i} \Delta_{i}^{j}(u) \quad j=1,2, \ldots
$$

where

$$
A^{j}(u)=\sum_{i=0}^{j-1}(-1)^{i} \frac{\Delta_{0}^{i}(u)}{2^{i+1}}
$$


expressing the ruin probability as an alternating series of forward-differences of arbitrary order $j,\left\{\Delta_{i}^{j}(u)\right\}_{i=0}^{\infty}$ also bound and smooth and $\lim _{i \rightarrow \infty} \Delta_{i}^{j}(u)=0$

Remark 1. The forward-differences are defined as

$$
\begin{aligned}
& \Delta_{i}^{j}(u)=\sum_{k=0}^{j}(-1)^{k}\left(\begin{array}{l}
j \\
k
\end{array}\right) a_{i+j-k}=\Delta_{i+1}^{j-1}(u)-\Delta_{i}^{j-1}(u) \quad j>0 \\
& \Delta_{i}^{0}(u)=a_{i}(u),
\end{aligned}
$$

Remark 2. The reader has probably realized that the terms $A^{j}(u)$ presented in the former Theorem are the successive approximations of the Euler acceleration technique, see for instance p. 131 of Press et al. (1986); just the very first term in the context of the approximations presented in this Theorem.

As a corollary of the former Theorem, we can consider the following family of approximations truncating (5.1), with explicit error terms

$$
\begin{aligned}
\Psi(u) & =S_{n}^{j}(u)+\varepsilon_{n}^{j}(u) \\
\Psi(u) & \simeq S_{n}^{j}(u)=A^{j}(u)+\left(\frac{(-1)^{j}}{2^{j}}\right) \sum_{i=0}^{n}(-1)^{i} \Delta_{i}^{j}(u) \\
n & =0,1,2, \ldots \quad j=1,2, \ldots
\end{aligned}
$$

It is easy to prove that this family of approximations can be obtained using the following alternative recursive formula

$$
S_{n}^{j}(u)=\frac{S_{n}^{j-1}(u)+S_{n+1}^{j-1}(u)}{2} ; \quad S_{n}^{0}(u) \equiv S_{n}(u)=\sum_{i=0}^{n} a_{i}(u)
$$

with a very positive effect on the loss of significant digits due to the substraction of small quantities compared with the former expression in which the forward-differences are directly involved. The formula used by Abate and Whitt (1995)

$$
E(m, n, u)=\sum_{k=0}^{m}\left(\begin{array}{c}
m \\
k
\end{array}\right) 2^{-m} S_{m+k}(u)
$$

can be considered less stable due to the increasing magnitude of the factorials involved in the calculations.

It is then obvious that the error can be expressed as an alternating series with bound and smooth terms and $\lim _{i \rightarrow \infty} \Delta_{i}^{j}(u)=0$

$$
\varepsilon_{n}^{j}(u)=\left(\frac{(-1)^{j}}{2^{j}}\right) \sum_{i=n+1}^{\infty}(-1)^{i} \Delta_{i}^{j}(u)
$$


The error magnitude will be decreasing with $j$ because

$$
\Delta_{i}^{j}(u)=h^{j} f^{j}\left(x_{i}\right)+O\left(h_{j}\right)
$$

where $f(x)$ is the original function which the original terms $a_{i}(u)$ come from and $x_{i}=x_{0}+h i$. We should remember that in the context of integration between zeros, see the former section,

$$
h \approx \frac{\pi}{u}
$$

reducing significantly the magnitude of the forward-differences, and subsequently the error terms as $j$ increases, when $u$ is large.

Remark 3. The value of the forward-differences, using the alternative formula (5.2), is obtained with the expression

$$
\left(\frac{(-1)^{j}}{2^{j}}\right)(-1)^{n} \Delta_{n}^{j}(u)=S_{n}^{j}(u)-S_{n-1}^{j}(u)
$$

In order to offer an estimation of the error, we can apply the same acceleration technique in the error term series and obtain

$$
\varepsilon_{n}^{j}(u)=\left(\frac{(-1)^{j+n+1}}{2^{j}}\right) \sum_{i=0}^{\infty}(-1)^{i} \frac{\Delta_{n+1}^{j+i}(u)}{2^{i+1}} \simeq\left(\frac{(-1)^{j+n+1}}{2^{j}}\right) \sum_{i=0}^{m}(-1)^{i} \frac{\Delta_{n+1}^{j+i}(u)}{2^{i+1}}
$$

Remark 4. This last formula offers a substantial improvement in the error estimation stated by Abate and Whitt (1995) in reference of Hosono (1984), where the following expression was used to assess the error

$$
\varepsilon_{n}^{j}(u) \simeq S_{n+1}^{j}(u)-S_{n}^{j}(u)=\frac{(-1)^{j+n+1} \Delta_{n+1}^{j}(u)}{2^{j}},
$$

the simplest case in our approach, when $m=1$.

Moreover, because of (5.3)

$$
\begin{gathered}
\left|\Delta_{n+1}^{j}(u)\right|>\left|\Delta_{n+1}^{j+1}(u)\right| \\
\operatorname{sign}\left(\varepsilon_{n}^{j}(u)\right) \simeq(-1)^{j+n+1} \Delta_{n+1}^{j}(u)
\end{gathered}
$$

and it is easy to deduce that the sign of the error terms, $\varepsilon_{n}^{j}(u)$ will be alternating for two consecutive values $(n, n+1)$ when

$$
\operatorname{sign}\left(\Delta_{n+1}^{j}(u)\right)=\operatorname{sign}\left(\Delta_{n+2}^{j}(u)\right)
$$

producing a sequence of upper and lower bounds for the ultimate ruin probability. One should bear in mind that this last condition is observed in most cases. 


\section{THE MID-POINT INTEGRATION ALGORITHM}

Let us now present a way to control the discretization total error avoiding a massive number of evaluations of the function $\operatorname{Re}\left(\Psi^{*}(c+z i)\right) \cos (u z)$ within the intervals $z \in\left(\frac{\pi}{2 u}+\frac{(k-1) \pi}{u}, \frac{\pi}{2 u}+\frac{\kappa \pi}{u}\right)$ for $k=1,2, \ldots$

The use of the mid-point integration rule with step size $h=\pi / 2 u$ and parameter $c=A / 2 u$ on the family of integrals $\left\{a_{k}(u)\right\}_{k=0}^{\infty}$ yields the expression

$$
\begin{aligned}
\Psi(u)= & \frac{e^{A / 2}}{2 u} \operatorname{Re}\left(\Psi *\left(\frac{A}{2 u}\right)\right) \\
& +\frac{e^{A / 2}}{u} \sum_{j=1}^{\infty}(-1)^{j} \operatorname{Re}\left(\Psi *\left(\frac{A}{2 u}+\left(\frac{j \pi}{u}\right) i\right)\right)
\end{aligned}
$$

Remark 5. The reader can easily realize that in this context the mid-point integretion is exactly the same as the three-point trapezoidal rule, and assign

$$
\begin{aligned}
& a_{0}(u)=\frac{e^{A / 2}}{2 u} \operatorname{Re}\left(\Psi^{*}\left(\frac{A}{2 u}\right)\right) \\
& a_{i}(u)=\frac{e^{A / 2}}{u} \operatorname{Re}\left(\Psi^{*}\left(\frac{A}{2 u}+\left(\frac{j \pi}{u}\right) i\right)\right) i=1,2, \ldots
\end{aligned}
$$

This approach is similar to the one stated in Abate and Whitt (1995), in reference of Dubner and Abate (1968), but obtained in the context of the Fourierseries method of Laplace transform inversion. Using this integration method, we only need the mid-point of each and every interval to approximate the family $\left\{a_{i}(u)\right\}_{i=0}^{\infty}$ with a dramatic reduction in the number of evaluations of the Laplace transform.

Moreover, Abate and Whitt (1995) also proved that the discretization error of the former expression can be controlled with the parameter $A$ and the formula

$$
\left|e^{d}(u)\right| \leq \frac{e^{-A}}{1-e^{-A}} \simeq e^{-A} \quad \text { (for } e^{-A} \text { small) }
$$

This last fact means, as stated by the mentioned authors, that in order to have at most $10^{-\gamma}$ discretization error $A=\gamma \log (10)$.

One should consider that the larger the value of $A$ (and smaller truncation error) the more accuracy is needed in the calculations of $\left\{\operatorname{Re}\left(\Psi^{*}\left(\frac{A+2 i \pi I}{2 t}\right)\right)\right\}_{i=0}^{\infty}$ because, to obtain the final approximation, the common factor $\frac{e^{4 / 2}}{i}$ will be used in (6.1). So the trade-off between significant digits used and discretization error becomes clear.

Unfortunately, the mentioned trade-off is not a new fact at all in numerical approximations; for a very clear example of this fact on approximating ruin probabilities see Usábel (1999). As a consequence, although much faster, 
when we use the mid-point integration, the algorithm can become unstable when searching for a high degree of accuracy and the significant digits are not upgraded.

Once more, dealing with large initial reserves, $u$, will be positive in the context of this methodology because of the above cited factor $\frac{e^{A / 2}}{u}$, avoiding the need of increasing the significant figures to perform the calculations.

\section{ASYMPTOTIC Formulas}

One of the main advantages of the method presented in this work is that it is not negatively affected by the size of the initial reserves considered as happened to be with other methods previously used in actuarial literature (see the introduction). For large values of $u$ most of the methods became either unstable or of a very slow convergence.

We cannot conclude this work without a mention to the main asymptotic approximations for ultimate ruin probability or the tail probabilities of the stationary waiting times, as its counterpart in queueing theory.

Considering a heavy-tailed service time or claim size distribution leads to the search of special formulae designed for these subexponential distributions. In the context of risk theory, Embrechts and Veraverbeke (1982) produced the formula

$$
\Psi(u) \sim \frac{\int_{u}^{\infty}(1-F(y)) d y}{\theta p_{1}} \quad u \rightarrow \infty
$$

Later Willekens and Teugels (1992) found a generalization of the above result including more terms in the final formula in the context of $M / G / 1$ queues

$$
\begin{gathered}
\Psi(u) \sim \\
+\frac{\int_{u}^{\infty}(1-F(y)) d y}{\theta p_{1}}+\left(\frac{\left(p_{1}\right)^{2}(1-\theta)}{p_{2} \theta^{2}}\right)(1-F(u)) \\
+\left(\frac{3\left(p_{1}\right)^{3}(1-\theta)^{2}}{4\left(p_{2}\right)^{2} \theta^{3}}+\frac{\left(p_{1}\right)^{2}(1-q)}{3 p_{3} \theta^{2}}\right) f(u)
\end{gathered}
$$

where

$$
p_{k}=\int_{0}^{\infty} x^{k} f(x) d x
$$

Unfortunately, the three terms approximations cannot be used for the heaviesttailed members of the family of the $\Gamma$-convolution claim sizes because the moments are not defined (see table 1 and 2) and the approximations in the numerical illustrations considered later are not so good as expected for very large initial reserves $u$.

Abate, Choudhury and Whitt (1994) also obtained an special formula for Pareto mixture of exponentials service time distributions. 


\section{Numerical Illustration}

We will consider as an illustration, Pareto claim sizes with c.d.f.

$$
F(x)=1-\left(\frac{\lambda}{\lambda+x}\right)^{\lambda+1} \quad \mathrm{x} \geq 0 \quad \lambda>0 \text { (integer) }
$$

as one of the heaviest tailed members of the family of $\Gamma$-convolutions claim size. The Laplace transforms of the example considered can be expressed in terms of the exponential integral (see for example Gradshteyn and Ryzhik (1994) formula 3.353.2).

We will find approximations for the rapidly oscillatory integral (3.2), the Bromwich-Mellin inversion formula, using the simple recursive expression (5.2) and the mid-point integration for the integrals (3.5)

$$
\begin{aligned}
& S_{n}^{j}(u)=\frac{S_{n}^{j-1}(u)+S_{n+1}^{j-1}(u)}{2} ; \quad S_{n}^{0}(u) \equiv S_{n}(u)=\sum_{i=0}^{n} a_{i}(u) \\
& a_{0}(u)=\frac{e^{A / 2}}{2 u} \operatorname{Re}\left(\Psi^{*}\left(\frac{A}{2 u}\right)\right) \\
& a_{i}(u)=\frac{e^{A / 2}}{u} \operatorname{Re}\left(\Psi^{*}\left(\frac{A}{2 u}+\left(\frac{j \pi}{u}\right) I\right)\right) \quad i=1,2, \ldots
\end{aligned}
$$

The value of the parameter $A$ was set so that the approximations had a discretization error of at most 15 significant digits $(A=15 \log (10))$, see section 6

TABLE 1

Pareto Claim SIZE $\lambda=1$

ULTIMATE RUIN PROBABILITY INTERVALS A $=15$ LOG(10)

\begin{tabular}{llll}
\hline \hline$\theta$ & \multicolumn{1}{c}{$u$} & \multicolumn{1}{c}{$\left(S_{9}^{20}(u), S_{10}^{20}(u), S_{11}^{20}(u)\right)$} & Asymptotic (one term) \\
\hline 0.1 & 1 & $(8.50144942,8.50144943) 10^{-1}$ & \\
& 10 & $(6.271279490,6.27179501) 10^{-1}$ & \\
100 & $(1.64859138,1.64859141) 10^{-1}$ & \\
1,000 & $(1.13443368,1.13443373) 10^{-2}$ & \\
10,000 & $(1.016661353,1.016661386) 10^{-3}$ & $9.999000110^{-4}$ \\
& 100,000 & $(1.00209834,1.00209837) 10^{-4}$ & $9.999900010^{-5}$ \\
$1,000,000$ & $(1.0002553,1.0002559) 10^{-5}$ & $9.999990010^{-6}$ \\
\hline 1 & & $(6.909906847,6.909906853) 10^{-1}$ & \\
& 10 & $(3.726769676,3.726769680) 10^{-1}$ & \\
& 100 & $(5.22265530,5.22265551) 10^{-2}$ & \\
& 1,000 & $(4.1948538,4.1948539) 10^{-3}$ & $3.99960010^{-4}$ \\
10,000 & $(4.0260816,4.0260817) 10^{-4}$ & $3.99996010^{-5}$ \\
100,000 & $(4.00332776,4.00332778) 10^{-5}$ & $3.99999610^{-6}$ \\
\hline
\end{tabular}


for details. Note that in Table 2 this value is upgraded to $201 \mathrm{n}(10)$ because the probability is very small.

The lower and upper bounds for the ultimate ruin probability will be spotted using the simple rule stated in the last paragraph of section 5. The approximations considered were $\left(S_{9}^{20}(u), S_{10}^{20}(u), S_{11}^{20}(u)\right)$ so that the maximum number of evaluations of the function $\operatorname{Re}\left(\Psi^{*}(c+z I)\right)$ is just 31$)$ !

The asymptotic approximations are based on the formula by Willekens and Teugels (1992), expression (7.1), considering the maximum number of terms possible depending on the value of $\lambda$.

TABLE 2

PARETO ClaIM SIZE $\lambda=2$

ULTIMATE RUIN PROBABILITY INTERVALS A $=15$ LOG(10)

\begin{tabular}{llll}
\hline \hline$\theta$ & \multicolumn{1}{c}{$u$} & \multicolumn{1}{c}{$\left(S_{9}^{20}(u), S_{10}^{20}(u), S_{11}^{20}(u)\right)$} & Asymptotic (two terms) \\
\hline 0.1 & 1 & $(8.41831695,8.41831696) 10^{-1}$ & \\
& 10 & $(5.22719526,5.22719527) 10^{-1}$ & \\
& 100 & $(1.8279697,1.8279700) 10^{-2}$ & \\
& 1,000 & $(4.3448088,4.3448093) 10^{-5}$ & $4.0001994010^{-7}$ \\
& 10,000 & $(4.0308031,4.0308034) 10^{-7}$ & $4.0000199910^{-9}$ \\
& 100,000 & $(4.0030442,4.0030445) 10^{-9}$ & \\
& $1,000,000$ & $*(4.00030,4.00036) 10^{-11}$ & \\
\hline 0.25 & 1 & $(6.760398370,6.760398375) 10^{-1}$ & \\
& 10 & $(2.522264643,2.522264644) 10^{-1}$ & \\
& 100 & $(2.4590058,2.4590063) 10^{-3}$ & $1.599600010^{-7}$ \\
& 1,000 & $(1.6478781,1.6478783) 10^{-5}$ & $1.599960010^{-9}$ \\
10,000 & $(1.645162,1.6045163) 10^{-7}$ & $1.599996010^{-11}$ \\
\hline 100,000 & $(1.6004484,1.6004485) 10^{-9}$ & \\
\hline
\end{tabular}

Remark 6. The intervals with the asterisk (*) were produced using $A=20 \ln (10)$ because of their small magnitude. An increase in the relative amplitude of the intervals in then observed.

It is very important to mention that the relative amplitude of the intervals (the relative error) is not quite affected when considering very large figures for the initial reserves, except in the cases mentioned in the remark above due to the change in the parameter $A$. All calculations were programmed in Maple V, release 4 using 22 significant digits.

\section{Concluding Comments}

The method presented in this work to approximate the ultimate ruin probability for $\Gamma$-convolutions claims size is specially recommended for large values of the initial reserves, $u$. As it is highlighted in sections 4, 5 and 6, considering 
even huge values for the initial reserves will not endanger either the accuracy or efficiency of this algorithm.

The general method of inverting Laplace transforms of tail probability distributions presented by Abate and Whitt (1995) is revisited in the context of the integration between zeros with three added improvements (see section 5 for details):

\section{A better error estimation}

2. The generation of upper and lower bounds.

3. A more stable recursive formula.

Consequently, the approach contemplated in this work is granted with the main advantages of the resursive methods based on discretization (see section 1) but not with their main drawback: the accuracy and efficiency is menaced considering large initial reserves. The reader should consider the accuracy obtained with just 31 evaluations of the Laplace transform and initial reserves $u=$ $1,000,000$ and calculations performed with 22 significant figures.

On the other side, the classical works of actuarial literature, mainly developed by the Scandinavian School in the 70's (see section 1), based their numerical illustrations on the Piessens' algorithms of inverting Laplace transforms (Piessens $(1969,71)$. In the survey of numerical methods for inverting the Laplace transform by Davis and Martin (1979), a clear conclusion can be reached: the error of the approximations was not easy to control.

Methods considered very good in this study, for instance Piessens and Branders (1971), can lead to poor results when dealing with increasing values of the initial reserves $u$. Seal (1975) already claimed that Laguerre series cannot be recommended as a practical method of numerical inversion of Laplace transform and the result can also affect to any other orthogonal polynomials. Piessens (1971, section 7, comment 2) also cited the limitation of Gaussian quadrature based methods for increasing accuracy demands. The method presented is a good alternative to the Gaver-Stehfest algorithm of inverting Laplace transforms, see Usábel (1999), because the demands of significant figures in the calculations are, by far, less restrictive. However, complex numbers are involved in the calculations.

Finally, the asymptotic formulae presented in section 8 did not yield, in the examples considered (see Table 1 and 2), so good approximations as expected for values of the initial reseves of magnitude $u=1,000,000$.

The algorithm was originally designed for $\Gamma$-convolution functions claim sizes because this family includes some of the most famous heavy-tailed distributions used in actuarial works, i.e. Pareto and log-normal. Nevertheless, the approach can be extended to any other claim size d.f. when

$$
\lim _{z \rightarrow \infty} \operatorname{Re}\left(f^{*}(c+z i)\right)=0 \quad c \geq 0
$$

that is usually observed in density functions with not very restrictive smoothness conditions as it is proved in Abate and Whitt (1995). 


\section{APPENDIX}

\section{Proof of Theorem 1}

Let us start proving that the limit for the ruin probability function for generalized $\Gamma$-convolutions claim size gets asymptotically close to 0

$$
\lim _{z \rightarrow \infty} \operatorname{Re}\left(\Psi^{*}(c+z I)\right)=0
$$

Abate and Whitt (1995) already showed that, under some restrictive smoothness conditions, a complementary c.d.f. fulfills

$$
\operatorname{Re}\left(\Psi^{*}(c+z I)\right)=\frac{c-\Psi^{\prime}(0)}{z^{2}}+o\left(z^{-2}\right)
$$

and obviously the asymptotic limit of the expression above.

However, we wanted to prove the same statement straight from the definition of $\Gamma$-convolutions claim size in order to offer a more complete proof for this type of distributions regardless extra considerations on smoothness. The following lemma shows the asymptotic behaviour for the Laplace transform of the d.f. of the $\Gamma$-convolution claim size distributions

Lemma 3. The Laplace transform of the density function of $\Gamma$-convolution function can be expressed

$$
f^{*}(c+z I)=R(c, z) \cos (\Theta(c, z))+I R(c, z) \sin (\Theta(c, z))
$$

where

$$
\begin{aligned}
& R(c, z)=e^{-a c} e^{-\frac{1}{2} \int_{0}^{\infty} \ln \left(1+2\left(\frac{c}{y}\right)+\left(\frac{c}{y}\right)^{2}+\left(\frac{z}{y}\right)^{2}\right) d U(y)} \\
& \Theta(c, z)=-a z-\int_{0}^{\infty} \arctan \left(\frac{z}{y+c}\right) d U(y)
\end{aligned}
$$

modulus and argument respectively, and

$$
\lim _{z \rightarrow \infty} f^{*}(c+z i)=0 \quad c \geq 0
$$

Proof. Let us expand the following natural logarithm

$$
\begin{aligned}
& \left.\ln \left(\frac{y}{y+c+z I}\right)=\ln (y)-\ln ((y+c)+z I)\right) \\
& =\ln (y)-\ln \left(\sqrt{(y+c)^{2}+z^{2}}\right)-\arctan \left(\frac{z}{y+c}\right) I
\end{aligned}
$$


and

$$
\begin{aligned}
e^{\int_{0}^{\infty} \ln \left(\frac{1}{1+\frac{y}{y}}\right) d U(y)}= & e^{\int_{0}^{\infty} \ln \left(\frac{y}{y+s}\right) d U(y)} \\
= & \left(e^{-\frac{1}{2} \int_{0}^{\infty} \ln \left(1+2\left(\frac{c}{y}\right)+\left(\frac{c}{y}\right)^{2}+\left(\frac{z}{y}\right)^{2}\right) d U(y)}\right) \\
& e^{I\left(-\int_{0}^{\infty} \arctan \left(\frac{z}{y+c}\right) d U(y)\right)}
\end{aligned}
$$

Using the definition (2.4)

$$
\begin{aligned}
f^{*}(s)= & e^{-a s} e^{\int_{0}^{\infty} \ln \left(\frac{1}{1+\left(\frac{s}{y}\right)}\right) d U(y)} \\
= & \left(e^{-a c-\frac{1}{2} \int_{0}^{\infty} \ln \left(1+2\left(\frac{c}{y}\right)+\left(\frac{c}{y}\right)^{2}+\left(\frac{z}{y}\right)^{2}\right) d U(y)}\right) \\
& e^{I\left(-a z-\int_{0}^{\infty} \arctan \left(\frac{z}{y+c}\right) d U(y)\right)} \\
= & R(c, z) \cos (\Theta(c, z))+\operatorname{IR}(c, z) \sin (\Theta(c, z))
\end{aligned}
$$

The last statement (9.3) follows from the limit

$$
\lim _{z \rightarrow \infty} \ln \left(1+2\left(\frac{c}{y}\right)+\left(\frac{c}{y}\right)^{2}+\left(\frac{z}{y}\right)^{2}\right)=\infty
$$

and

$$
\lim _{z \rightarrow \infty} \int_{0}^{\infty} \ln \left(1+2\left(\frac{c}{y}\right)+\left(\frac{c}{y}\right)^{2}+\left(\frac{z}{y}\right)^{2}\right) d U(y)=\infty
$$

where $U(y)$ is nondecreasing $\left(U^{\prime}(y) \geq 0\right)$ using the definition of generalized $\Gamma$ convolution functions. Finally, using (9.2) and (9.1)

$$
\lim _{z \rightarrow \infty} R(c, z)=0 \Rightarrow \lim _{z \rightarrow \infty} f^{*}(c+z l)=0
$$

Let us now finally prove that, for $\Gamma$-convolution function claim size, the Laplace transform of the ruin probability function will be asymptotically close to zero as $z$ increases in any event.

If we expand expression (2.3) using the results previously obtained from the former lemma (9.3)

$$
\Psi^{*}(c+z I)=\frac{1}{c+z I}-\frac{\left(\frac{\theta}{1+\theta}\right)}{(c+z I)-\left(\frac{I}{(1+\theta) p_{1}}\right)}
$$

and just focusing on the real part

$$
\begin{aligned}
& \lim _{z \rightarrow \infty} \operatorname{Re}\left(\Psi^{*}(\dot{c}+z \nearrow)\right) \\
= & \lim _{z \rightarrow \infty} \frac{c}{c^{2}+z^{2}}-\frac{\theta\left((1+\theta) p_{1} c-1\right)}{\left((1+\theta) p_{1} c-1\right)^{2}+\left((1+\theta) p_{1} z\right)^{2}}=0
\end{aligned}
$$

proving statement 1 of Theorem 1 . 
The real part of the Laplace transform of the ruin probability function

$$
\begin{aligned}
\Psi^{*}(c+z I)= & \int_{0}^{\infty} e^{-(c+z l) x} \Psi(x) d x \\
= & \int_{0}^{\infty} e^{-c x} \cos (-z x) \Psi(x) d x \\
& +\left(\int_{0}^{\infty} e^{-c x} \sin (-z x) \Psi(x) d x\right) I
\end{aligned}
$$

can be bound using the absolute value convergence Theorem for integrals

$$
\int_{0}^{\infty}\left|e^{-c x} \cos (-z x) \Psi(x)\right| d x \leq \int_{0}^{\infty} e^{-c x} \Psi(x) d x
$$

The last integral will be always convergent for non-negative $c$ because $\Psi(x)$ is a decreasing function and

$$
\lim _{x \rightarrow \infty} \Psi(x)=0
$$

and it is clear that we cannot find any singularities of $\Psi^{*}(s)$ when $c$ is nonnegative, proving that it is bound and smooth.

\section{Proof of Theorem 2}

If the initial sequence $\left\{a_{i}(u)\right\}_{i=0}^{\infty}$ is bound and smooth and $\lim _{i \rightarrow \infty} a_{i}=0$, the forward-differences defined as

$$
\begin{aligned}
& \Delta_{i}^{j}(u)=\sum_{k=0}^{j}(-1)^{k}\left(\begin{array}{l}
j \\
k
\end{array}\right) a_{i+j-k}(u)=\Delta_{i+1}^{j-1}(u)-\Delta_{i}^{j-1}(u) \quad j>0 \\
& \Delta_{i}^{0}(u)=a_{i}(u)
\end{aligned}
$$

will be also bound and smooth and decreasing with $i, \lim _{i \rightarrow \infty} \Delta_{i}^{j}(u)=0$.

Remark 7. the simplified notation $a_{i}$ and $A_{i}^{j}$ will be used henceforth. The alternating initial series can be easily modified into

$$
\begin{aligned}
\Psi(u) & =\sum_{i=0}^{\infty}(-1)^{i} a_{i} \\
& =\frac{a_{0}}{2}-\frac{\left(a_{1}-a_{0}\right)}{2}+\frac{\left(a_{2}-a_{1}\right)}{2}-\ldots \\
& =\frac{a_{0}}{2}-\frac{\Delta_{0}^{1}}{2}+\frac{\Delta_{1}^{1}}{2}-\ldots \\
& =\frac{a_{0}}{2}-\sum_{i=0}^{\infty}(-1)^{i} \frac{\Delta_{i}^{1}}{2}
\end{aligned}
$$


and the same procedure can be applied to the new alternating series obtained $\sum_{i=0}^{\infty}(-1)^{i} \frac{\Delta_{i}^{1}}{2}$. If we continue applying the same easy transform recursively to the new alternating series in terms of the forward-differences the expression (5.1) results.

\section{ACKNOWLEDGMENTS}

I am indebted to the anonymous referees of this paper for their useful comments, specially on bibliography, that lead to a substantial improvement of the previous version of this work.

\section{REFERENCES}

ABATE, J. and WhiTt, W. (1992) The Fourier-series method for inverting transforms of probability distributions. Queueing Systems 10, 5-88.

ABATE, and WhITT, W. (1995) Numerical inversion of Laplace transforms of probability distributions. ORSA Journal on Computing 7, No. 1, 36-43.

ABATE, and WHITT, W. (1995) An operational calculus for probability distributions via Laplace Transforms. Advances in Applied Probability, 28, 75-113.

Abate, J., Choudhury, G. and Whitt, W. (1994) Waiting-time tail probabilities in queues with long-tail service-time distribution. Queueing Systems, 16, 311-338.

Abate, J., Choudhury, G. and WhitT, W. (1995) Calculating the M/G/1 busy period density and LIFO waiting-time distribution by direct numerical transform inversion. Operations Research Letters 18, 113-119.

AbRAMOWITZ, M. and STEGUn, I.A. (1972) Handbook of Mathematical Functions. Ninth Edition. New York, N.Y. Dover Publications.

BERG, C. (1981) The Pareto distribution is a generalized $\Gamma$-convolution - a new proof. Scan. Act. Journal, 117-119.

BOAS, R. (1987) Invitation to complex analysis. The Random House/Birkhäuser mathematics series. New York.

Bohman, H. (1971) Ruin probabilities. Skandinavisk Aktuarietidskrift, 159-163.

BoHman, H. (1974) Fourier Inversion-Distribution functions-Long tails. Scand. Actuarial Journal, 43-45.

Bohman, H. (1975) Numerical inversion of characteristic functions. Scan. Act. Journal, 121-124.

Bowers, N.L., Gerber, H.U., Hickman, J.C., Jones, D.A. and Nesbith, C.J. (1997) Actuarial Mathematics. Society of Actuaries.

CAI, J. and GARrido, J. (1998) Aging properties and bounds for ruin probabilities and stop-loss premiums. Insurance: Mathematics and Economics 23, Number 1, 33-44.

Choudhury, M.L., Gupt, U.C., Agarwal, M. (1992) Exact and approximate numerical solutions to steady-state single-server queues: M/G/1 - a unified approach. Queueing Systems 10, 351-380.

CoudhuRY, M.L. and WhITT, W. (1997) Scaling for Numerical Transform Inversion to analyze Teletraffic Models. ITC 15, 933-942.

Conway, J. (1978) Functions of one complex variable I. Second edition. Springer-Verlag. New York.

Cramèr, H. (1955) Collective Risk Theory. Jubille Volume of F. Skandia.

DAVIES, B. and MARTIN, B. (1979) Numerical inversion of the Laplace transform: a survey and comparison of methods. Journal of computational physics 33, 1-32.

DAVIES, J.D. and RABINOWITZ, P. (1984) Methods of numerical Integration. Second edition. Academic Press Inc. Boston. 
DICKSON, D.C.M. (1989) Recursive calculation of the probability and severity of ruin. Insurance: Mathematics and Economics 8, 145-148.

Dickson, D.C.M., Egidio dos Reis, A.D., Waters, H.R. (1995) Some Stable Algorithms in Ruin Theory and their Application. ASTIN Bulletin 25, 153-175.

Dickson, D.C.M. and WATERS, H.R. (1991) Recursive calculation of survival probabilities. ASTIN Bulletin 21, 199-221.

Embrechts, P. and Veraverbeke, N. (1982) Estimates for Probability of Ruin with special emphasis on the possibility of large claims. Insurance: Mathematics and Economics 1, 55-72.

GERBER, H.U. (1979) An Introduction to Mathematical Risk Theory. Huebner Foundation Monograph 8. Philadelphia, Pa., University of Pennsylvania.

Gerber, H. GoovaerTs, M., KAAs, R. (1987) On the probability and severity of ruin. ASTIN Bulletin 17, n. 2, 151-163.

GLYNN, P. and WhITT, W. (1995) Heavy-traffic extreme-value limits for queues. Operations Research Letters 18, 107-111.

Goovaerts, M. D'Hooge, L., De PrIL, N. (1977) On a class of generalized $\Gamma$-convolutions. Scan. Act. Journal, 21-30.

GoovaerTs, M. and DE VyLder, F. (1984) A Stable Recursive Algorithm for Evaluation of Ultimate Ruin Probabilities. ASTIN Bulletin 14, 53-59.

Gradshteyn, I.S. and Ryzhik, I.M. (1994) Table of Integrals, Series and Products. Fifth edition. Academic Press.

Heckman, P. and Meyers, G. (1983) The calculation of aggregate loss distributions from claim severity and claim count distributions. Proceedings of the Casuality Actuarial Society LXX, 22-61.

Hosono, T. (1984) Fast Inversion of the Laplace Transform by Basic. Kyoritsu Publishers, Japan.

JOYCE, D.C. (1971) Survey of extraolation processes in numerical analysis. SI AM Rev. 13, 435490.

Kendall, M. and Stuart, A. (1977) The Advanced Theory of Statistics. Vol. I, 4th Edition. MacMillan.

LEvin, D. (1973) Development of non-linear transformations for improving convergence of sequences. Internat. J. Comput. Math. 3, 371-388.

LONGMAN, I.M. (1956) Note on a method for computing infinite integrals of oscillatory functions. Proc. Cambridge Philos. Soc. 52, 764-768.

Panjer, H.H. (1986) Direct Calculation of Ruin Probabilities. Journal of Risk and Insurance 53, $521-529$.

PANJER, H.H., WANG, S. (1993) On the Stability of recursive Formulas. ASTIN Bulletin 23, 227258.

PANJer, H.H., Willmot, G.E. (1992) Insurance risk Models. Society of Actuaries, Schaumburg.

PIESSENS, R. (1969) New quadrature formulas for the numerical inversion of Laplace transforms. BIT 9, 351-361.

Piessens, R. (1971) Gaussian Quadrature Formulas for the Numerical Integration of Bromwich's integral and the Inversion of the Laplace Transform. Journal of Engineering Math. 5, $1-5$.

Piessens R. and Branders, M. (1971) Numerical Inversion of the Laplace Transform using generalised Laguerre Polynomials. Proceedings of the IEE 118, No. 10.

Ramsay, C.M. (1992a) A Practical Algorithm for Approximating the Probability of Ruin. Transactions of the Society of Actuaries XLIV, 443-59.

RaMSAY, C.M. (1992b) Improving Goovaerts' and De Vylder's Stable Recursive Algorithm. ASTIN Bulletin 22, 51-59.

RamsaY, C.M. and UsÁBel, M.A. (1997). Calculating Ruin probabilities via Product integration. ASTIN Bulletin 27, n. 2, 263-271.

SEAL, H. (1971) Numerical calculation of the Bohman-Esscher family of convolution-mixed negative binomial distribution functions. Mitt. Verein. schweiz. Versich.-Mathr. 71, 71-94.

SEAL, H. (1974) The numerical calculation of $U(w, t)$, the probability of non-ruin in an interval $(0, t)$. Scan. Act. Journal, 121-139.

SEAL H.L. (1975) A note on the use of Laguerre polynomials in the inversion of Laplace transforms. Blätter der DAVM 12, 131-134. 
SEAL, H. (1977) Numerical inversion of characteristic functions. Scan. Act. Journal, 48-53.

THORIN, O. (1970) Some remarks on the ruin problem in case the epochs of claims form a renewal process. Skandinavisk Aktuarietidskrift, 29-50.

THORIN, O. (1971) Further remarks on the ruin problem in case the epochs of claims form a renewal process. Skandinavisk Aktuarietidskrift, 14-38, 121-142.

THORIN, $O$. (1973) The ruin problem in case the tail of a distribution is completely monotone. Skandinavisk Aktuarietidskrift, 100-119.

THORIN, O. (1977) Ruin probabilities prepared for numerical calculation. Scandinavian Actuarial Journal.

Thorin, O. (1977) On the infinite divisibility of the Pareto distribution. Scan. Act. Journal, 3140.

Thorin, O. (1977) On the infinite divisibility of the Lognormal distribution. Scan. Act. Journal, 121-148.

Thorin, O. (1978) An extension of the notion of a generalized $\Gamma$-convolution. Scan. Act. Journal, 141-149.

THORIN, O. and WiKSTAD, N. (1973) Numerical evaluation of ruin probabilities for a finite period. ASTIN Bulletin VII:2, 138-153.

USÁBEL, M. (1999) Calculating multivariate ruin probabilities via Gaver-Stehfest inversion technique. IME 25, 133-142.

VAN DE VOOREN, A.I. and VAN LINDE, H.J. (1966) Numerical calculation of integrats with strongly oscillating integrand. Math. Comp., 232-245.

WIKSTAD, N. (1971) Exemplifications of ruin probabilities. ASTIN Bulletin 6, part 2.

WiKSTAD, N. (1977) How to calculate Ruin probabilities according to the classical Risk Theory. Scand. Actuarial Journal.

WILlekens, E. and TeugELS, J.L. (1992) Asymptotic expansions for waiting time probabilities in a M/G/1 with long-tailed service time. Queueing Systems 10, 295-312.

Miguel A. UsÁBEL

Universidad Carlos III de Madrid

Avda. Universidad Carlos, 22

Colmenarejo 28270 (Madrid)

Spain

E-mail: usabel@emp.uc3m.es 